\title{
A NEW NONDETERMINISTIC METHOD FOR OPTIMAL SELECTION OF MASTER DEGREES OF FREEDOM FOR DYNAMIC CONDENSATION BASED ON EVOLUTIONARY OPTIMIZATION
}

\author{
Waldemar Mucha \\ Silesian University of Technology, Department of Computational Mechanics and Engineering, Gliwice, Poland \\ e-mail: waldemar.mucha@polsl.pl
}

\begin{abstract}
The following paper presents a new method for choosing a set of master degrees of freedom for the process of dynamic condensation in order to reduce a finite element model. The general rule is that the more degrees of freedom are eliminated, the more accurate the reduced model is. However, eliminating different subsets (of equal sizes) of degrees of freedom may influence the accuracy differently. Therefore, choosing an optimal subset is crucial. The presented method is based on multicriterial evolutionary optimization which makes it the first nondeterministic approach based on computational optimization technique for this application.
\end{abstract}

Key words: dynamic condensation, optimization, evolutionary algorithm, model order reduction

\section{Introduction}

Discretization of a high-complexity or large-scale mechanical system often results in a high order numerical model (that is described by a large number of ordinary differential equations). In other words, finite element (FE) discretization of sophisticated mechanical systems may result in too many degrees of freedom (DOFs) to perform time-effective and accurate computations. In such cases, a low-order approximation of the high-order model can be found (Antoulas, 2005; Chaturantabut and Sorensen, 2010; de Klerk et al., 2008). Such low order approximations are particularly useful when computations are performed repeatedly and FE system matrices are constant, e.g. in computation of the system response to a certain excitation in the time domain (structural dynamics, where computations are performed step-after-step), when solving an inverse problem (like optimization or identification, where many direct problems must be solved to obtain a solution to an inverse problem) or computation of frequency response functions (Bai, 2002; Besselink et al., 2013). One of model order reduction techniques applied to FE models is condensation. It consists of eliminating a subset of DOFs from the model. The eliminated DOFs are referred to as slaves, while the remaining DOFs are called masters ( $\mathrm{Qu}, 2013)$. The condensation technique was proposed for the first time by Guyan and Irons (Guyan, 1965). The Guyan condensation is also referred to as static condensation. The numerical procedure is quite simple, however inertia effects are ignored, therefore, the reduced model gives accurate results only to static problems. Dynamic condensation techniques do not have this drawback, the inertia effects of slave DOFs are partially or entirely taken into consideration in the final model. Different approaches on how to perform dynamic condensation can be found in the literature (Chen and Pan, 1988; Leung, 1979; Mokeyev, 1998; Qu, 2013; Suarez and Singh, 1992). Most commonly used are iterative methods for dynamic condensation where the initial reduced model is set, and in every subsequent iteration the system matrices are modified in order to reduce the error of the model (Friswell et al., 1995, 1998; Qu, 2013; Qu and Fu, 2000; Suarez and Singh, 
1992). The crucial part of performing dynamic condensation is to choose a priori a subset of DOFs to be kept as masters because choosing different subsets results in drastically different accuracy of reduced models. Not only the number of chosen DOFs matters, but also which of them are chosen. A new nondeterministic method for optimal choice of masters, based on evolutionary optimization approach, is presented in the paper.

Apart from possible applications of the reduced models described above, another motivation for optimal model order reduction is real-time application of the finite element method (FEM), e.g. hybrid simulation (Drazin and Govindjee, 2017; Mucha, 2019; Mucha and Kuś, 2017; Ramos et al., 2016), surgery simulations (Audette et al., 2004; Berkley et al., 2004; Lapeer et al., 2010) and control of elastic soft robots (Duriez, 2013). In such cases, the finite element model must be reduced as much as possible, while remaining required accuracy, in order to enable or speed-up real-time computations where the calculation time is crucial. The computational effort to prepare the reduced model is irrelevant as long as the efficiency of real-time computations increases, because in such applications often every millisecond matters. Section 2 describes the mechanism of condensation. In Section 3, the known methods for master DOFs selection are summarized and the new method is presented in details. Section 4 consists of two numerical examples comparing the results of applying the presented method with the results obtained from the most popular known method for master DOFs choice. In Section 5, the results are discussed and the conclusion is presented.

\section{Model order reduction using iterative methods of dynamic condensation}

In structural dynamics, the equation of motion of a mechanical system is usually written as a set of linear second-order differential equations in the matrix form

$$
\mathbf{M} \ddot{\mathbf{u}}(t)+\mathbf{C} \dot{\mathbf{u}}(t)+\mathbf{K u}(t)=\mathbf{F}(t)
$$

where $\mathbf{M}, \mathbf{C}$ and $\mathbf{K}$ are the mass, damping and stiffness matrices, respectively, $\ddot{\mathbf{u}}, \dot{\mathbf{u}}$ and $\mathbf{u}$ are the acceleration, velocity and displacement vectors, respectively, $\mathbf{F}$ is the vector of exciting forces and $t$ means time (Zienkiewicz and Taylor, 2000).

In order to perform the condensation, all the DOFs must be divided a priori to masters (indicated by subscript $m$ ) and slaves (subscript $s$ )

$$
\begin{aligned}
& {\left[\begin{array}{cc}
\mathbf{M}_{m m} & \mathbf{M}_{m s} \\
\mathbf{M}_{s m} & \mathbf{M}_{s s}
\end{array}\right]\left[\begin{array}{c}
\ddot{\mathbf{u}}_{m}(t) \\
\ddot{\mathbf{u}}_{s}(t)
\end{array}\right]+\left[\begin{array}{ll}
\mathbf{C}_{m m} & \mathbf{C}_{m s} \\
\mathbf{C}_{s m} & \mathbf{C}_{s s}
\end{array}\right]\left[\begin{array}{c}
\dot{\mathbf{u}}_{m}(t) \\
\dot{\mathbf{u}}_{s}(t)
\end{array}\right]} \\
& +\left[\begin{array}{cc}
\mathbf{K}_{m m} & \mathbf{K}_{m s} \\
\mathbf{K}_{s m} & \mathbf{K}_{s s}
\end{array}\right]\left[\begin{array}{c}
\mathbf{u}_{m}(t) \\
\mathbf{u}_{s}(t)
\end{array}\right]=\left[\begin{array}{c}
\mathbf{F}_{m}(t) \\
\mathbf{F}_{s}(t)
\end{array}\right]
\end{aligned}
$$

In order to perform reduction of Eq. (2.1), the system matrices and force vector are transformed as follows

$$
\mathbf{M}_{\mathbf{R}}=\mathbf{T}^{\mathrm{T}} \mathbf{M} \mathbf{T} \quad \mathbf{C}_{\mathbf{R}}=\mathbf{T}^{\mathrm{T}} \mathbf{C} \mathbf{T} \quad \mathbf{K}_{\mathbf{R}}=\mathbf{T}^{\mathrm{T}} \mathbf{K} \mathbf{T} \quad \mathbf{F}_{\mathbf{R}}(t)=\mathbf{T}^{\mathrm{T}} \mathbf{F}(t)
$$

where $\mathbf{T}$ means the transformation matrix. $\mathbf{R}$ is the dynamic condensation matrix. It is computed in an iterative scheme of dynamic condensation. In the literature, different iterative methods can be found, for example (Friswell et al., 1995; $\mathrm{Qu}$ and $\mathrm{Fu}, 2000$ ). The relation between $\mathbf{T}$ and $\mathbf{R}$ is

$$
\mathbf{T}=\left[\begin{array}{l}
\mathbf{I} \\
\mathbf{R}
\end{array}\right]
$$

where $\mathbf{I}$ is the identity matrix $(\mathrm{Qu}, 2013)$. 
After transformation, equation (2.1) takes the form

$$
\mathbf{M}_{\mathbf{R}} \ddot{\mathbf{u}}_{m}(t)+\mathbf{C}_{\mathbf{R}} \dot{\mathbf{u}}_{m}(t)+\mathbf{K}_{\mathbf{R}} \mathbf{u}_{m}(t)=\mathbf{F}_{\mathbf{R}}(t)
$$

If damping of the model is proportional, with Rayleigh coefficients $\alpha$ and $\beta$

$$
\mathbf{C}=\alpha \mathbf{M}+\beta \mathbf{K}
$$

the damping matrix of the reduced model is also proportional to the reduced mass and stiffness matrices

$$
\mathbf{C}_{\mathbf{R}}=\alpha \mathbf{M}_{\mathbf{R}}+\beta \mathbf{K}_{\mathbf{R}}
$$

\section{Selection of master degrees of freedom}

Let the set of all numbered DOFs be denoted as $\mathbf{D}$ (of length $n$ ) and the subset of master DOFs chosen for condensation (equation (2.2)) be denoted as $D_{r, j}$. The subscript describes the number of master DOFs (size of the subset) as $r$ and $j$-th combination of the subset choice, where $j=1,2, \ldots, c$ and

$$
c=\frac{n !}{r !(n-r) !}
$$

If the accuracy of the reduced model (Eq. (2.5)) is described by an error coefficient err (determining the magnitude of differences in the results obtained by the reduced and accurate model), then err will be a function of the size of the reduced model and the chosen combination of DOFs to be masters

$$
e r r=f(r, j)
$$

The selection of master degrees of freedom has usually a significant influence on the accuracy of the reduced model. The general rule is that the more master DOFs are selected, the higher accuracy is obtained. However, the accuracy of reduced models based on different sets of master DOFs (of equal size) may be drastically different. Therefore, a right choice of masters is crucial.

\subsection{State of the art}

The known algorithms for choosing a set of DOFs to be kept as masters can be divided into qualitative and quantitative methods.

Qualitative methods are, in fact, more guidelines than algorithms. They are based on simple heuristic rules that an engineer should follow based on his experience. For complex mechanical systems, it can be unintuitive and difficult. Ramsden and Stoker (1969) advice to keep DOFs of the most flexible areas with the highest concentration of mass. Levy (1971) suggests keeping the DOFs that are associated to the largest values of mass or the largest values in eigenvectors. On the other hand, in (Popplewell et al., 1973) the authors choose the DOFs with the highest possible strain energy.

Quantitative algorithms allow one to perform automatic selection of a set of DOFs. Most of these methods are based on the ratio kii/mii (where kii and mii are the i-th diagonal elements of stiffness and mass matrix, respectively) (Henshell and Ong, 1974; Matta, 1987; Ong, 1987; Shah and Raymund, 1982; Suarez and Singh, 1992). Bouhaddi and Filod (1992) proved that these algorithms give poor results if the mass is not uniformly distributed in the mechanical system. In the literature, there can also be found algorithms based on so-called modal energy associated with DOFs in mode shapes approximated by Ritz vectors (Kim and Choi, 2000; Li, 2003). 
The research (Downs, 1980; Kim and Choi, 2000; Li, 2003) shows that usually rotational DOFs should be classified as slaves. If the mass matrix is lumped, eliminating all the massless rotational DOFs does not have an influence on accuracy. If the mass matrix is consistent, rotational DOFs matter only for frequencies corresponding to higher eigenvalues.

\subsection{Evolutionary optimization approach}

As it was mentioned, the accuracy of a reduced model is not only dependent on its size but also on a particular combination of the choice of master DOFs. The proposed algorithm is probably the first that answers both questions: (i) how many degrees of freedom and (ii) which to choose as masters in order to maximize the accuracy of the reduced model. The aforementioned algorithms found in the literature only answer the second question, and the answer to the first one is usually based on user's experience who makes an a priori assumption of constant $r$.

The new approach to select the master DOFs utilizes a multicriterial evolutionary algorithm with binary coding. Evolutionary algorithms are a group of nondeterministic algorithms for optimization that are inspired by mechanisms of biological evolution of species. In evolutionary algorithms, potential solutions to a given problem are called individuals, the objective function evaluates adaptation of individuals to environment which is determined by the optimized problem. The individuals exchange genetic material between each other (by crossover operators) and random changes occur in the genes of individuals (mutation operators) - this way new individuals are created. From the potential solutions, the biggest chance to survive have those mostly adapted to the environment (Beluch and Długosz, 2016; Jin, 2011; Mrozek et al., 2015).

The motivation to utilize multicriterial evolutionary algorithm with binary coding in the considered application consists of three reasons. The first is that binary coding is natural for the considered problem - every DOF must be classified as a master or slave. Therefore, the number of design variables is equal to the number of DOFs of the mechanical system. Algorithms with floating-point coding could also be used, however introducing corrections to artificially adjust floating-point variables to represent binary values is too much computationally expensive and might be not so efficient. The second reason is that the result of optimization is a set of not dominated solutions, therefore, the user can choose the most suitable for his/her requirements of the accuracy and number of DOFs. The last reason is that evolutionary algorithms have good immunity to local minima. In papers (Burczyński et al., 2015; Poteralski et al., 2014) evolutionary algorithms are compared to particle swarm algorithms, and it turns out that evolutionary algorithms have significantly greater immunity to local minima compared to particle swarm algorithms (and it is suspected that the considered problems may have a great number of local optimums), although swarm algorithms tend to converge faster.

The optimization is multicriterial - with two criteria

$$
r \rightarrow \min
$$

and

$$
e r r=\sum_{i=1}^{k}\left|\frac{\omega_{i, R}-\omega_{i}}{\omega_{i}}\right| \rightarrow \min
$$

First criterion (3.3) is the minimal number of master DOFs, which is trivial. Second criterion (3.4) is the maximum accuracy which is numerically achieved by minimization of error function (3.2). The error function is represented by the sum of relative errors of the first $k$ eigenvalues, where $\omega_{i}$ is the $i$-th eigenvalue of the full model, and $\omega_{i, R}$ is the $i$-th eigenvalue of the reduced model. In order to determine the eigenvalues, the eigenproblem must be solved

$$
\mathrm{K} \Phi=\Omega^{2} \mathrm{M} \Phi
$$


where $\boldsymbol{\Phi}$ is the matrix of eigenvectors and $\boldsymbol{\Omega}$ is the diagonal matrix of eigenvalues $\omega_{i}$. For the reduced problem, the eigenproblem is solved similarly, by inputting the reduced stiffness and mass matrices

$$
\mathbf{K}_{\mathbf{R}} \Phi_{\mathbf{R}}=\Omega_{\mathbf{R}}^{2} \mathbf{M}_{\mathbf{R}} \Phi_{\mathbf{R}}
$$

The utilized evolutionary algorithm is the well-known method NSGA-II (Deb et al., 2002). The algorithm uses the elitist principle (the elites of population have the biggest opportunity to survive and be carried to the next generation) and an explicit diversity preserving mechanism (crowding distance). NSGA-II emphasizes the non-dominated solutions.

The proper choice of the number of considered eigenvalues $n$ is very important. A heuristic rule is proposed where the frequency related to the $k$-th eigenvalue must be at least three times higher than the maximum frequency $\omega_{\max }$ that will affect the reduced model by excitation forces

$$
\omega_{k} \geqslant 3 \omega_{\max }
$$

This way the reduced model will be optimized to have the maximum accuracy in the whole range of excitation forces frequency.

A constraint must be introduced in the process of optimization - the number of DOFs of each individual must not be smaller than $k$. This can be achieved by applying the penalty approach the fitness function values of both criteria $(r$ and $e r r)$ increase at least two orders of magnitude when the constraint is exceeded.

The author recommends the following parameters of the evolutionary algorithm: tournament selection, uniform crossover with probability about 0.8 , mutation probability between 0.1 and 0.2. Two alternative stop conditions are advised: (i) maximum number of iterations (at least 100 times the number of binary design variables) and (ii) geometric average of the relative change in the value of spread over stall_gen generations is less than spread_tolerance, and the final spread is less than the mean spread over the last stall_gen generations. The spread is defined as

$$
\text { spread }=\frac{g+s}{g+2 s}
$$

where $s$ denotes the standard deviation of the proximity of individuals lying on the Pareto front to the nearest neighbor, and $g$ is the sum over two objective function criteria of the norm of the difference between the current minimum-value Pareto point for that index and the minimum point for that index in the previous iteration. Such stopping criteria guarantee that the algorithm stops when the extreme objective function values do not change significantly over subsequent iterations (variable $g$ ) and when the points on the Pareto front are spread evenly (variable $s$ ). The number of individuals in population, stall_gen and spread_tolarance should be set by trial and error. The values set in the numerical examples gave good results.

It is recommended to plot the Pareto front after performing the optimization. It allows one to intuitively choose a solution which will give the required accuracy and size of the reduced model.

The presented method applies especially to full models with several dozens or several hundreds of DOFs because of its computational cost of optimization (number of design variables) and eigenproblem solution. For a bigger number of DOFs, it is recommended to utilize iterative eigensolvers that compute only the first $k$ eigenvalues.

If the mass matrix is lumped, then all the massless DOFs (rotational) should be classified a priori as slaves to reduce the order of the optimization problem. The engineering practice shows that even if the mass matrix is consistent, the rotational DOFs should be, in most cases, removed in the process of condensation. 


\section{Numerical examples}

Two numerical examples are provided that compare the effectiveness of the proposed algorithm with the popular known algorithm - Matta's scheme.

\subsection{Numerical example 1 - two beams with a spring}

The first numerical example concerns a mechanical system, presented in Fig. 1, that consists of two beams and a spring that joins them. The cross-section of the beams is a circular pipe. The outside and inside diameters on the thicker end are 50 and $40 \mathrm{~mm}$, respectively. The diameters on the narrower end are 30 and $25 \mathrm{~mm}$, respectively. The total length of both beams is $2 \mathrm{~m}$, the spring joins them in half-length. The beams are made of aluminum (Young modulus $70 \mathrm{GPa}$, density $2720 \mathrm{~kg} / \mathrm{m}^{3}$ ). The spring is characterized by stiffness of $400 \mathrm{~N} / \mathrm{mm}$ and mass of $0.6 \mathrm{~kg}$. The finite element model consists of 21 finite elements (20 beam elements and one bar element representing the spring). The system has got 39 DOFs. For future reference, the DOFs will be determined by a combination of the node number and letter $\mathrm{V}$ (indicating vertical DOF) or $\mathrm{R}$ (indicating rotational DOF).

(a)

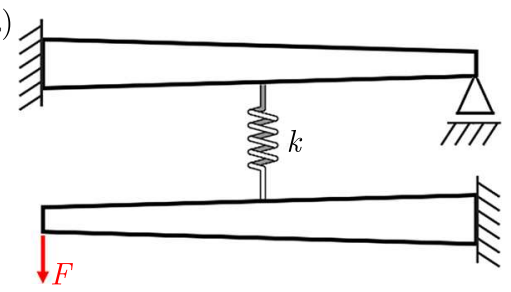

(b)

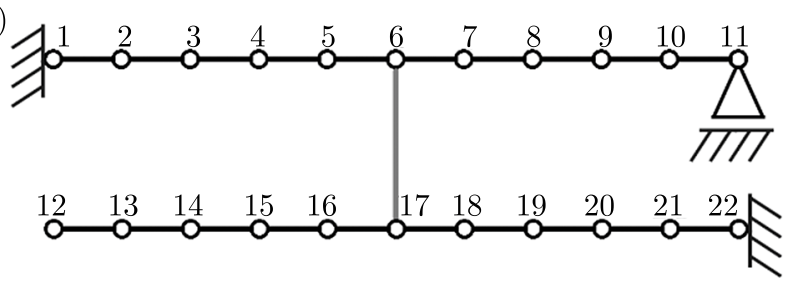

Fig. 1. Numerical example 1: (a) mechanical system, (b) numerical model

The utilized system of consistent units is: $\mathrm{N}, \mathrm{mm}, \mathrm{MPa}, \mathrm{ms}, \mathrm{g}$. The mass matrix is consistent. The damping matrix is proportional, according to Eq. (2.6), with coefficients $\alpha=2$ and $\beta=0.1$. The first 8 eigenfrequencies of the model are listed in Table 1.

Table 1. Eigenfrequencies of the system - numerical example 1

\begin{tabular}{|c|c|c|c|}
\hline $\begin{array}{c}\text { Eigenvalue } \\
\text { number }\end{array}$ & $\begin{array}{c}\text { Frequency } \\
{[\mathrm{Hz}]}\end{array}$ & $\begin{array}{c}\text { Eigenvalue } \\
\text { number }\end{array}$ & $\begin{array}{c}\text { Frequency } \\
{[\mathrm{Hz}]}\end{array}$ \\
\hline \hline 1 & 20.72 & 5 & 173.92 \\
\hline 2 & 50.83 & 6 & 282,34 \\
\hline 3 & 116.41 & 7 & 338.47 \\
\hline 4 & 154.50 & 8 & 512.24 \\
\hline
\end{tabular}

It is assumed that the maximum frequency of the exciting force is $450 \mathrm{~Hz}$. Therefore, the number of eigenfrequencies $k$ considered in error function (3.2) should not be less than 4 in the following example. The minimal value is set in the analyses: $k=4$.

The evolutionary optimization of the master DOFs set, according to the presented algorithm, was performed with the following parameters: 400 individuals in population, tournament selection with size 2, scattered crossover fraction 0.8 , uniform mutation fraction 0.1. The parameters of stopping criteria are: maximum number of iterations 4100 , stall_gen $=100$, spread_tolerance $=10^{-4}$. The stop condition was met after 123 generations.

The constraint that every individual must have at least 4 DOFs was introduced using the penalty approach.

The Pareto front of the performed optimization is presented in Fig. 2. For future considerations, 3 individuals were chosen: with $4(3 \mathrm{~V}, 5 \mathrm{~V}, 6 \mathrm{~V}, 12 \mathrm{~V}), 6(6 \mathrm{~V}, 12 \mathrm{~V}, 13 \mathrm{~V}, 14 \mathrm{~V}, 18 \mathrm{~V}, 19 \mathrm{~V})$ and $8(2 \mathrm{~V}, 3 \mathrm{~V}, 5 \mathrm{~V}, 6 \mathrm{~V}, 8 \mathrm{~V}, 19 \mathrm{~V}, 14 \mathrm{~V}, 19 \mathrm{~V})$ DOFs. 


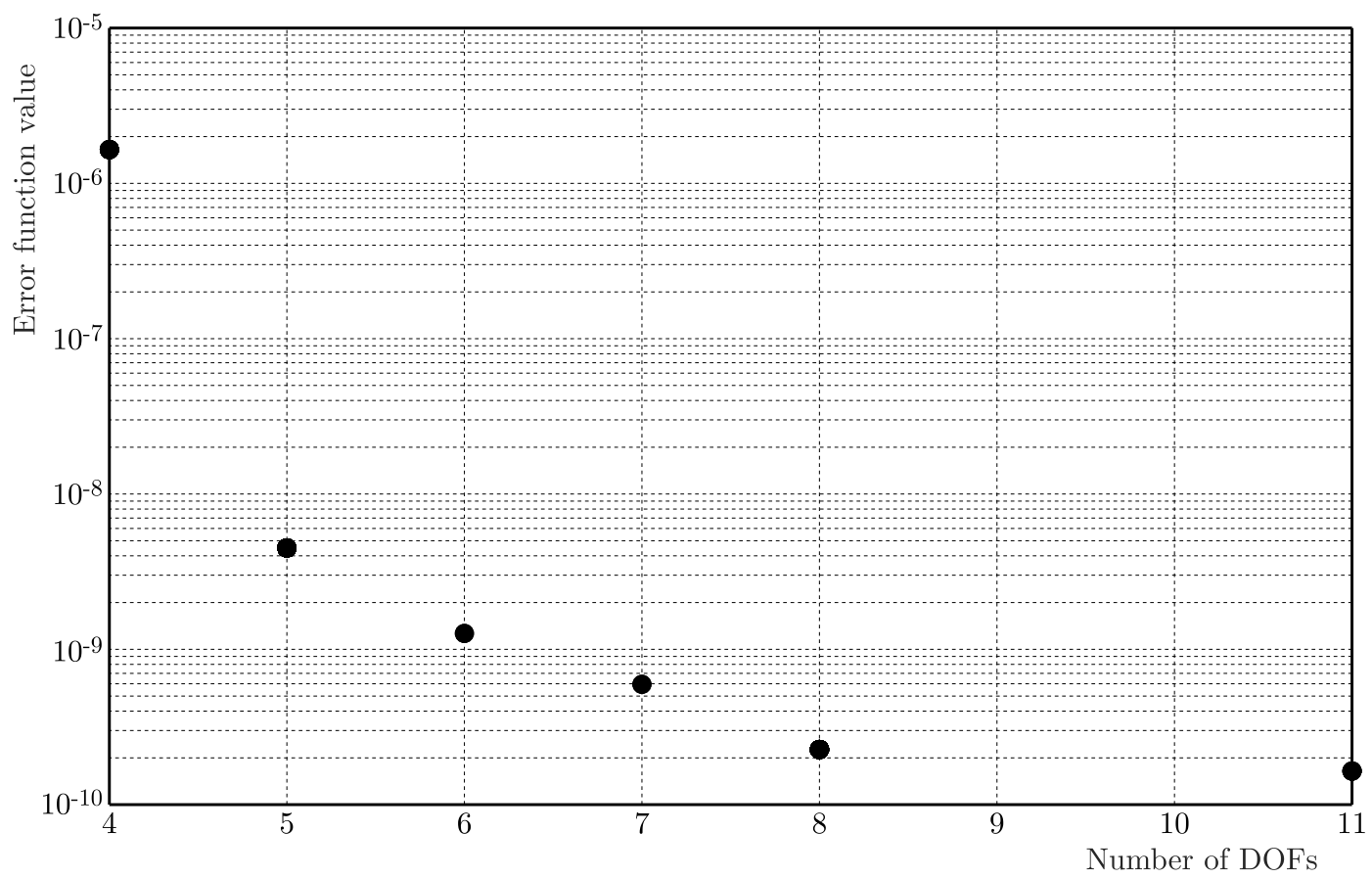

Fig. 2. Pareto front for numerical example 1

The mechanical system was reduced to the same number of DOFs using Matta's scheme (Matta, 1987). The 4 DOFs acquired from Matta's scheme were: $6 \mathrm{~V}, 12 \mathrm{~V}, 15 \mathrm{~V}, 17 \mathrm{~V}$. Analogously the 6 DOFs are: $6 \mathrm{~V}, 8 \mathrm{~V}, 12 \mathrm{~V}, 15 \mathrm{~V}, 17 \mathrm{~V}, 20 \mathrm{~V}$. The 8 DOFs are: $3 \mathrm{~V} 6 \mathrm{~V}, 8 \mathrm{~V}, 10 \mathrm{~V}, 12 \mathrm{~V}, 15 \mathrm{~V}$, $17 \mathrm{~V}, 20 \mathrm{~V}$.

As one can see, both algorithms classified the rotational DOFs as slaves (although consistent mass matrices were utilized), which complies with the known rules of engineering practice.

Table 2. Error function values for different solutions of evolutionary approach and Matta's scheme - numerical example 1

\begin{tabular}{|c|c|c|}
\hline Number of DOFs & Evolutionary approach & Matta's scheme \\
\hline \hline 4 & $1.65 \cdot 10^{-6}$ & $1.40 \cdot 10^{-1}$ \\
\hline 6 & $1.27 \cdot 10^{-9}$ & $1.10 \cdot 10^{-8}$ \\
\hline 8 & $2.26 \cdot 10^{-10}$ & $4.91 \cdot 10^{-9}$ \\
\hline
\end{tabular}

Table 2 compares the error function values for solutions including 4, 6 and 8 DOFs chosen by evolutionary approach and Matta's scheme. As one can see, in every case the proposed algorithm results with a smaller error than in Matta's scheme. An exemplary dynamical analysis was performed. Dynamic condensation was performed using the method described in $(\mathrm{Qu}$ and $\mathrm{Fu}$, 2000). Newmark's implicit integration scheme was utilized, with the time step $1 \mathrm{~ms}$. The plot of force $F(t)$ is presented in Fig. 3. The frequency of the force varies from 0 to $50 \mathrm{~Hz}$.

A vertical displacement of node 6 was observed in the full and reduced models. The mean-squared errors of all reduced models are compared in Table 3. Plots of the vertical displacement of node 6, obtained from the full and reduced models, are presented in Fig. 3. For better visibility, only models reduced to 4 DOFs by Matta's scheme and evolutionary approach were considered. The plot labeled as the exact result is the numerical solution of the full model.

As one can see in Table 3 and Fig. 3, the proposed algorithm for the optimal master DOFs selection resulted in more accurate reduced models than in Matta's scheme. 
(a)

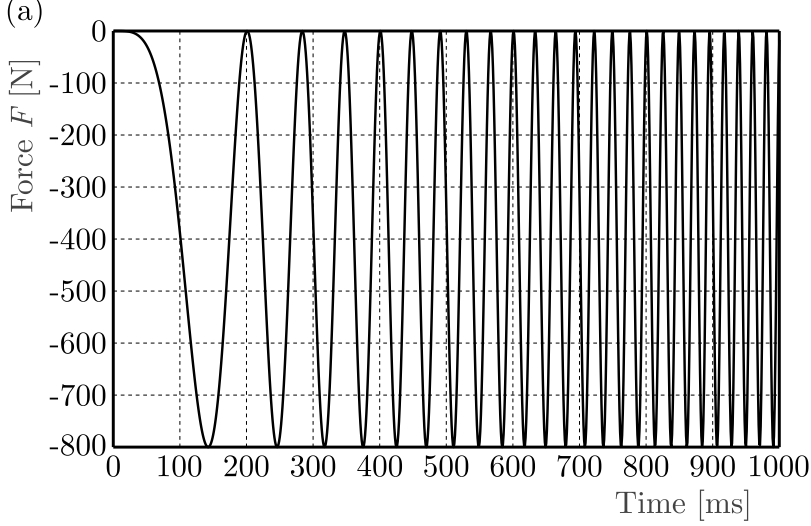

(b)

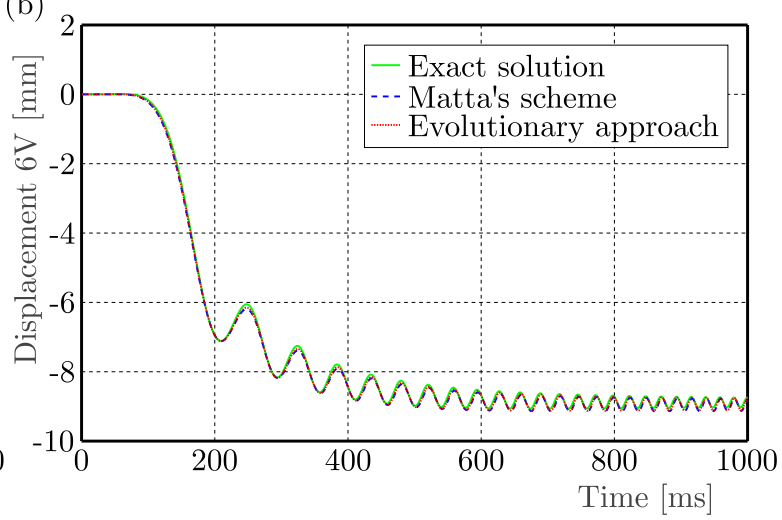

Fig. 3. Time courses: (a) force $F(t)$, (b) displacement $6 \mathrm{~V}$ for the full model and models reduced to 4 DOFs

Table 3. Mean-squared error between displacement $6 \mathrm{~V}$ in dynamical analysis of the full and reduced models $\left[\mathrm{mm}^{2}\right]$ - numerical example 1

\begin{tabular}{|c|c|c|}
\hline Number of DOFs & Evolutionary approach & Matta's scheme \\
\hline \hline 4 & $3.40 \cdot 10^{-3}$ & $7.01 \cdot 10^{-3}$ \\
\hline 6 & $6.23 \cdot 10^{-3}$ & $6.94 \cdot 10^{-3}$ \\
\hline 8 & $4.51 \cdot 10^{-5}$ & $5.56 \cdot 10^{-5}$ \\
\hline
\end{tabular}

\subsection{Numerical example 2 - bicycle frame}

The second numerical example is the frame of a mountain bicycle, originally described in (Mucha and Kuś, 2018), where it was an example of hybrid (hardware-in-the-loop) simulation in which FEM computations were performed in real time. Model order reduction for such applications may be very important. A photograph and CAD model of the frame is presented in Fig. 4. The material of the frame is steel (Young modulus $200 \mathrm{GPa}$, density $7800 \mathrm{~kg} / \mathrm{m}^{3}$ ).

(a)

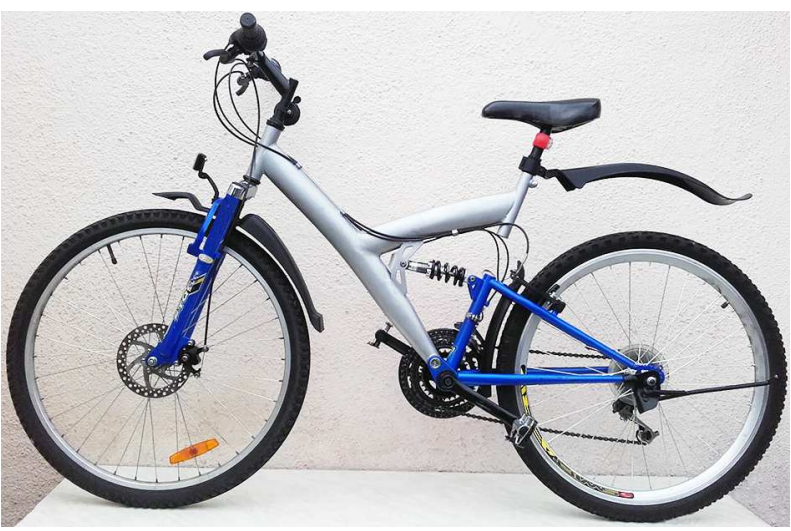

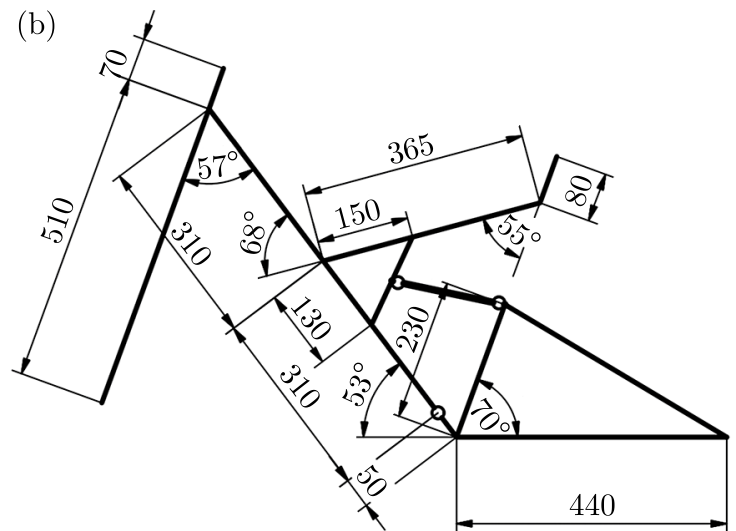

Fig. 4. Numerical example 2 - mountain bicycle: (a) photograph, (b) CAD model (dimensions in mm). Reprinted from (Mucha and Kuś, 2018), with the permission of AIP Publishing

The finite element model is presented in Fig. 5. It consists of 27 finite elements (numbered in circles), 25 nodes (numbered using grey font) and 73 DOFs. Element 17 represents the shock absorber of the bicycle and is modeled using a rod element with stiffness $131.35 \mathrm{~N} / \mathrm{mm}$. In node 18 , there is a ball joint, therefore, an extra rotational DOF was added - rotations on both sides of the node are not compatible. For future reference, the DOFs will be determined by combination of the node number and letter $\mathrm{H}$ (indicating horizontal DOF), $\mathrm{V}$ (indicating vertical 
$\mathrm{DOF}$ ) or $\mathrm{R}$ (indicating rotational DOF). The cross-section parameters of all other elements are presented in Table 4.

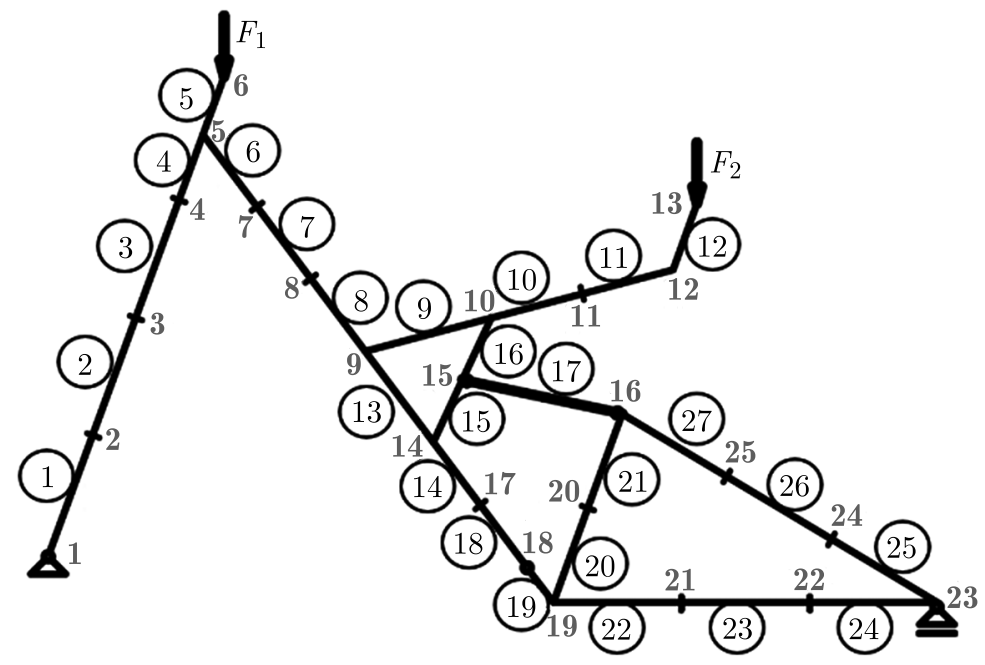

Fig. 5. Numerical example 2 - finite element model

Table 4. Cross-section parameters of numerical example 2. Reprinted from (Mucha and Kuś, 2018), with the permission of AIP Publishing

\begin{tabular}{|c|c|c|}
\hline Element number & Area $\left[\mathrm{mm}^{2}\right]$ & Moment of inertia $\left[\mathrm{mm}^{4}\right]$ \\
\hline \hline $1-3$ & 287 & 33507 \\
\hline $4-5$ & 158 & 22190 \\
\hline $6-8,13$ & 221 & 92112 \\
\hline $9-11$ & 231 & 104925 \\
\hline 12 & 130 & 12287 \\
\hline 14 & 210 & 75663 \\
\hline $15-16$ & 210 & 36463 \\
\hline 18 & 172 & 35695 \\
\hline 19 & 540 & 91125 \\
\hline $20-21$ & 134 & 13674 \\
\hline $22-27$ & 174 & 7508 \\
\hline
\end{tabular}

The utilized system of consistent units is: $\mathrm{N}, \mathrm{mm}, \mathrm{MPa}, \mathrm{ms}, \mathrm{g}$. The mass matrix is lumped. The damping matrix is proportional, according to Eq. (2.6), with coefficients $\alpha=2$ and $\beta=0.1$. The first 8 eigenfrequencies of the model are listed in Table 5 .

Table 5. Eigenfrequencies of the system - numerical example 2

\begin{tabular}{|c|c|c|c|}
\hline $\begin{array}{c}\text { Eigenvalue } \\
\text { number }\end{array}$ & $\begin{array}{c}\text { Frequency } \\
{[\mathrm{Hz}]}\end{array}$ & $\begin{array}{c}\text { Eigenvalue } \\
\text { number }\end{array}$ & $\begin{array}{c}\text { Frequency } \\
{[\mathrm{Hz}]}\end{array}$ \\
\hline \hline 1 & 20.77 & 5 & 453.38 \\
\hline 2 & 34.00 & 6 & 491.73 \\
\hline 3 & 297.58 & 7 & 547.67 \\
\hline 4 & 384.65 & 8 & 1149.60 \\
\hline
\end{tabular}

It is assumed that the maximum frequency of the exciting force is $150 \mathrm{~Hz}$. Therefore, the number of eigenfrequencies $k$ considered in error function (3.2) should not be less than 5 in the following example. The minimal value is set in the analyses $k=5$. 
All the rotary DOFs were classified a priori as slaves, DOFs $6 \mathrm{~V}$ and $13 \mathrm{~V}$ were classified a priori as masters. Therefore, the number of design variables was reduced to 45 . The constraint that every individual must have at least 5 DOFs was introduced using the penalty approach as in numerical example 1.

The evolutionary optimization parameters were the same as in the previous example.

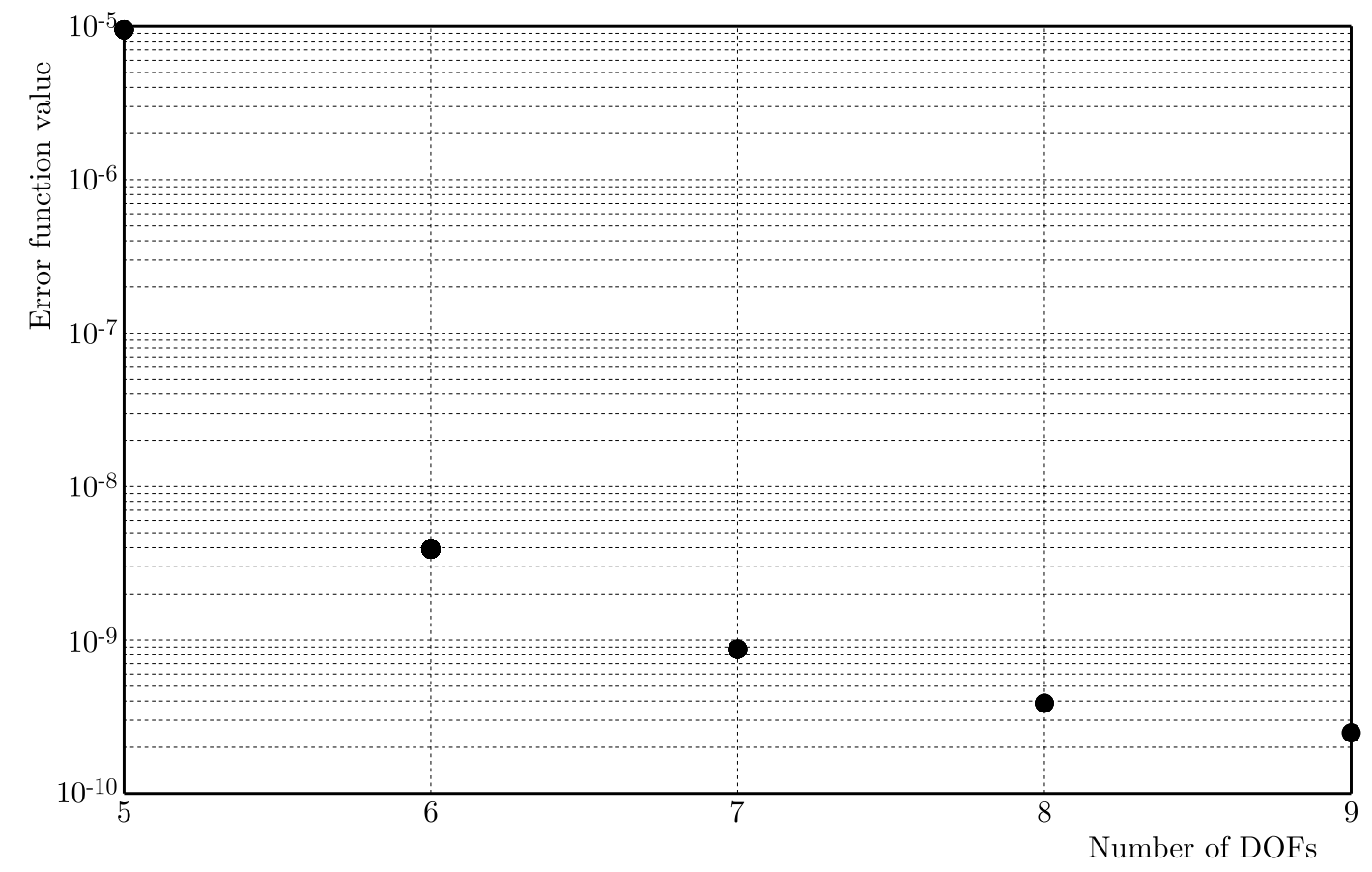

Fig. 6. Pareto front for numerical example 2

The Pareto front of the performed optimization is presented in Fig. 6. For future considerations, 3 individuals were chosen: with $5(6 \mathrm{~V}, 12 \mathrm{~V}, 13 \mathrm{~V}, 16 \mathrm{~V}, 20 \mathrm{~V}), 7(6 \mathrm{~V}, 12 \mathrm{~V}, 13 \mathrm{~V}, 15 \mathrm{~V}, 16 \mathrm{~V}$, $24 \mathrm{H})$ and $9(6 \mathrm{~V}, 9 \mathrm{~V}, 11 \mathrm{~V}, 12 \mathrm{~V}, 13 \mathrm{~V}, 19 \mathrm{~V}, 20 \mathrm{~V}, 23 \mathrm{H}, 24 \mathrm{H}) \mathrm{DOFs}$. The mechanical system was reduced to the same number of DOFs using Matta's scheme. The 5 DOFs acquired from Matta's scheme were: $3 \mathrm{H}, 5 \mathrm{~V}, 6 \mathrm{~V}, 13 \mathrm{~V}, 18 \mathrm{H}$. Analogously, the $7 \mathrm{DOF}$ are: $3 \mathrm{H}, 5 \mathrm{H}, 5 \mathrm{~V}, 6 \mathrm{~V}, 13 \mathrm{~V}, 18 \mathrm{H}$, $19 \mathrm{~V}$. The 9 DOFs are: $3 \mathrm{H}, 5 \mathrm{H}, 5 \mathrm{~V}, 10 \mathrm{~V}, 6 \mathrm{~V}, 13 \mathrm{~V}, 18 \mathrm{H}, 19 \mathrm{~V}, 25 \mathrm{~V}$.

Table 6. Error function values for different solutions of evolutionary approach and Matta's scheme - numerical example 2

\begin{tabular}{|c|c|c|}
\hline Number of DOFs & Evolutionary approach & Matta's scheme \\
\hline \hline 5 & $9.49 \cdot 10^{-6}$ & $5.16 \cdot 10^{-2}$ \\
\hline 7 & $8.70 \cdot 10^{-10}$ & $8.28 \cdot 10^{-9}$ \\
\hline 9 & $2.48 \cdot 10^{-10}$ & $9.91 \cdot 10^{-9}$ \\
\hline
\end{tabular}

Table 6 compares values of the error function for solutions including 4, 6 and 8 DOFs chosen by the evolutionary approach and Matta's scheme. As in the previous example, in every case, the proposed algorithm gives results with a significantly smaller error than in Matta's scheme.

An exemplary dynamical analysis was performed. The condensation was performed using an iterative method described in ( $\mathrm{Qu}$ and $\mathrm{Fu}, 2000)$. Newmark's implicit integration scheme was utilized, with the time step $1 \mathrm{~ms}$. The time of analysis was $20 \mathrm{~s}$. The frequency of forces $F_{1}$ and $F_{2}$ sweeped lineary from 0 to $150 \mathrm{~Hz}$. The plot of exciting forces $F_{1}(t)$ and $F_{2}(t)$ is presented in Fig. 7 (in the range 0-2 s, in the whole length it would be illegible). 


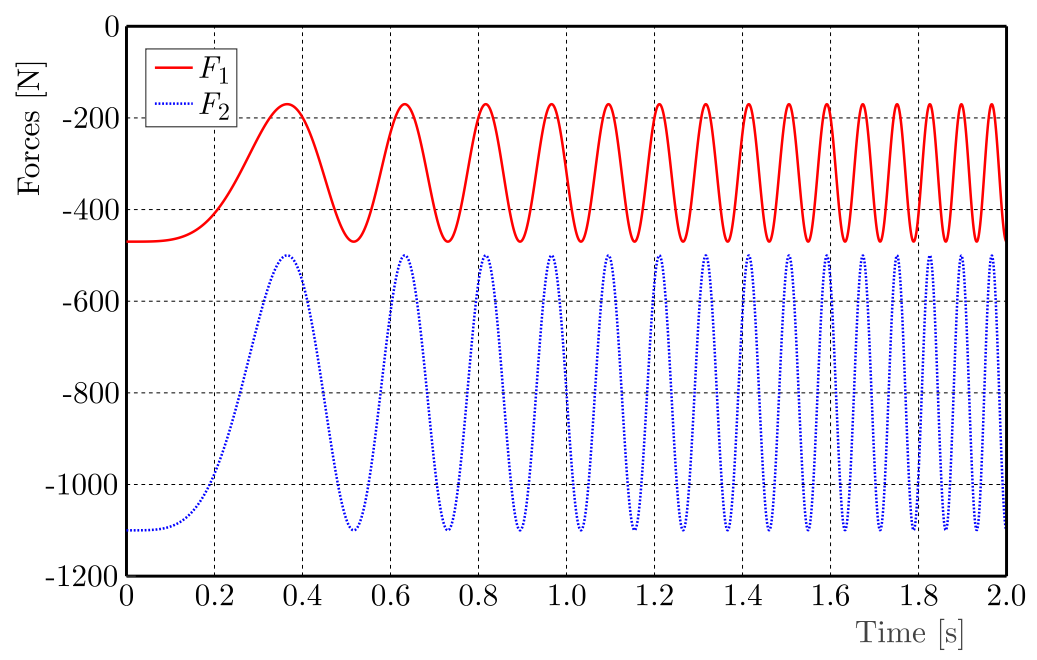

Fig. 7. Plot of the forces $F_{1}(t)$ and $F_{2}(t)$ in the range $0-2 \mathrm{~s}$

The vertical displacement of node 13 was observed in the full and reduced models. The mean-squared errors of all reduced models are compared in Table 7. Plots of the vertical displacement of node 13, obtained from the full (labeled as the exact result) and reduced models, are presented in Fig. 8. Only the models reduced to 5 DOFs by Matta's scheme and the evolutionary approach were taken into consideration in the plot.

Table 7. Mean-squared error between displacement 13V in dynamical analysis of the full and reduced models $\left[\mathrm{mm}^{2}\right]$ - numerical example 2

\begin{tabular}{|c|c|c|}
\hline Number of DOFs & Evolutionary approach & Matta's scheme \\
\hline \hline 5 & $1.40 \cdot 10^{-3}$ & $1.05 \cdot 10^{-2}$ \\
\hline 7 & $3.42 \cdot 10^{-4}$ & $3.43 \cdot 10^{-4}$ \\
\hline 9 & $5.44 \cdot 10^{-5}$ & $1.37 \cdot 10^{-4}$ \\
\hline
\end{tabular}
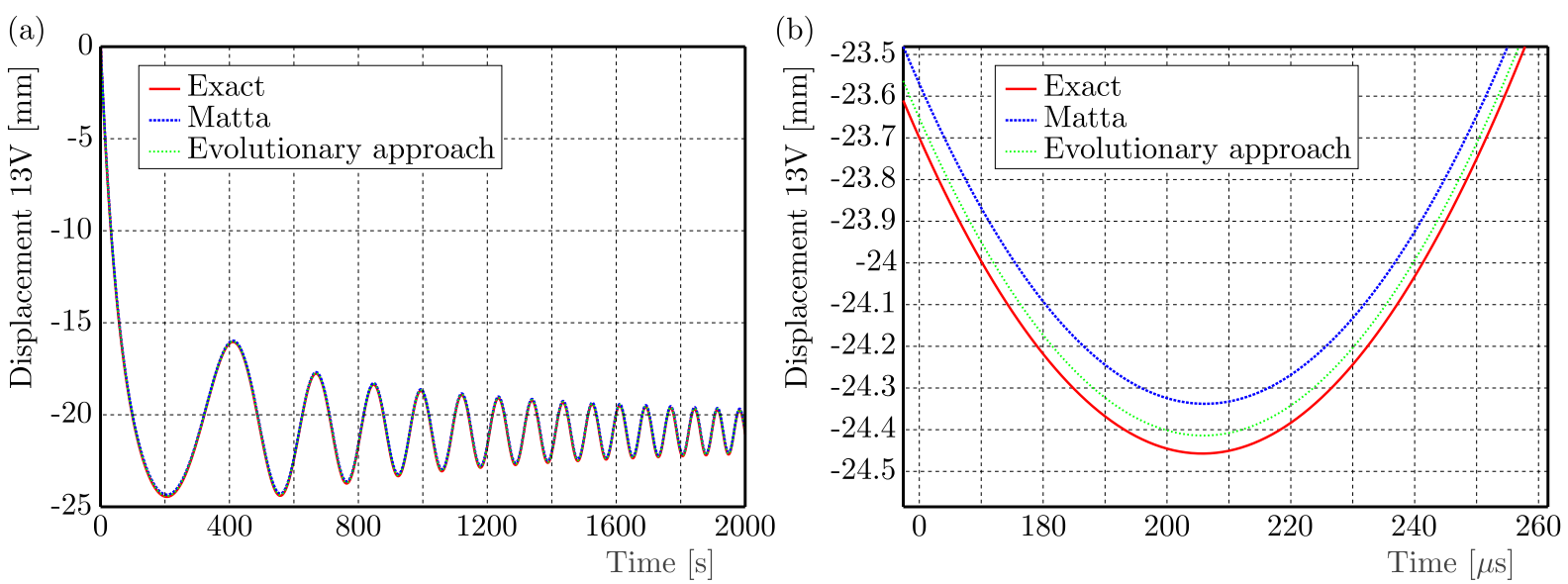

Fig. 8. Displacement 13V of the full model and models reduced to 5 DOFs: (a) in the range 0-2 s, (b) close-up on a fragment

As one can see in Table 7 and Fig. 8, the proposed algorithm for optimal master DOFs selection allowed one to obtain a more accurate model for reduction to every considered numbered of DOFs, compared to Matta's scheme. 


\section{Conclusion}

The paper presents a new method for optimal selection of master DOFs in order to perform model order reduction using the dynamic condensation technique. The accuracy of the reduced model depends on the number of DOFs that will be classified as masters, as well as it depends on which DOFs will become masters. The proposed algorithm is based on multicriterial evolutionary optimization, where the accuracy of the reduced model is maximized and the number of master DOFs is minimized. Such an approach has got a significant advantage compared to other previously-known methods - it allows one to relieve the user from choosing which and how many DOFs should be kept as masters. Unlike in other algorithms, the user obtains a set of possible solutions from which he/she can plot a Pareto front and choose one individual that meets the requirements of accuracy and the number of DOFs.

The presented algorithm is computationally demanding, however, as proven by numerical examples, it is very effective. The effectiveness was compared to Matta's scheme, which is used very often, and the proposed method resulted with a more accurate model for every size of the master subset.

Taking into account computing power of an average modern PC, implementing the proposed algorithm to mechanical systems with several hundred or several thousand DOFs should not be problematic.

One of the most important potential applications of the presented method is model order reduction for real-time applications, for example hybrid (hardware-in-the-loop) simulations using a real-time FEM. In such applications, the maximum reduction of the model (while maintaining the highest possible accuracy) is crucial. For many applications, all the computational effort made to reduce the model offline does not matter as long as it allows one to reduce the time step in real-time simulations by $1 \mathrm{~ms}$.

\section{Acknowledgement}

The research was partially funded from the statutory subsidy of the Faculty of Mechanical Engineering, Silesian University of Technology.

\section{References}

1. Antoulas A.C., 2005, An overview of approximation methods for large-scale dynamical systems, Annual Reviews in Control, 29, 181-190, DOI: 10.1016/j.arcontrol.2005.08.002

2. Audette M.A., Hayward V., Astley O., Doyon M., McCallister G.A., Chinzei K., 2004, A PC-based system architecture for real-time finite element-based tool-specific surgical simulation, International Congress Series, 1268, 378-383, DOI: 10.1016/j.ics.2004.03.294

3. BAI Z., 2002, Krylov subspace techniques for reduced-order modeling of large-scale dynamical systems, Applied Numerical Mathematics, 43, 9-44, DOI: 10.1016/S0168-9274(02)00116-2

4. Beluch W., DŁugosz A., 2016, Multiobjective and multiscale optimization of composite materials by means of evolutionary computations, Journal of Theoretical and Applied Mechanics, 54, 397-409, DOI: 10.15632/jtam-pl.54.2.397

5. Berkley J., Turkiyyah G., Berg D., Ganter M., Weghorst S., 2004, Real-time finite element modeling for surgery simulation: an application to virtual suturing, IEEE Transactions on Visualization and Computer Graphics, 10, 314-325, DOI: 10.1109/TVCG.2004.1272730

6. Besselink B., Tabak U., Lutowska A., Wouw N. van de Nijmeijer H., Rixen D.J., Hochstenbach M.E., Schilders W.H.A., 2013, A comparison of model reduction techniques from structural dynamics, numerical mathematics and systems and control, Journal of Sound and Vibration, 332, 4403-4422, DOI: 10.1016/j.jsv.2013.03.025 
7. Boumaddi N., Fillod R., 1992, A method for selecting master DOF in dynamic substructuring using the Guyan condensation method, Computers and Structures, 45, 941-946, DOI: 10.1016/00457949(92)90052-2

8. Burczyński T., Poteralski A., Szczepanik M., 2015, Immune and swarm optimization of structures, [In:] Advances in Evolutionary and Deterministic Methods for Design, Optimization and Control in Engineering and Sciences, D. Greiner, B. Galván, J. Périaux, N. Gauger, K. Giannakoglou, G. Winter (Eds.), Springer International Publishing, Cham, 295-308, DOI: 10.1007/9783-319-11541-2_19

9. Chaturantabut S., Sorensen D.C., 2010, Nonlinear model reduction via discrete empirical interpolation, SIAM Journal on Scientific Computing, 32, 2737-2764, DOI: 10.1137/090766498

10. Chen S.-H., Pan H.H., 1988, Guyan reduction, Communications in Applied Numerical Methods, 4, 549-556, DOI: $10.1002 / \mathrm{cnm} .1630040412$

11. De Klerk D., Rixen D.J., Voormeeren S.N., 2008, General framework for dynamic substructuring: history, review and classification of techniques, AIAA Journal, 46, 1169-1181, DOI: $10.2514 / 1.33274$

12. Deb K., Pratap A., Agarwal S., Meyarivan T., 2002, A fast and elitist multiobjective genetic algorithm: NSGA-II, IEEE Transactions on Evolutionary Computation, 6, 182-197, DOI: 10.1109/4235.996017

13. Downs B., 1980, Accurate reduction of stiffness and mass matrices for vibration analysis and a rationale for selecting master degrees of freedom, Journal of Mechanical Design, 102, 412-416, DOI: $10.1115 / 1.3254760$

14. Drazin P.L., Govindjee S., 2017, Hybrid simulation theory for a classical nonlinear dynamical system, Journal of Sound and Vibration, 392, 240-259, DOI: 10.1016/j.jsv.2016.12.034

15. Duriez C., 2013, Control of elastic soft robots based on real-time finite element method, [In:] 2013 IEEE International Conference on Robotics and Automation. Presented at the 2013 IEEE International Conference on Robotics and Automation, 3982-3987, DOI: 10.1109/ICRA.2013.6631138

16. Friswell M.I., Garvey S.D., Penny J.E.T., 1995, Model reduction using dynamic and iterative IRS technique, Journal of Sound and Vibration, 186, 311-323

17. Friswell M.I., Garvey S.D., Penny J.E.T., 1998, The convergence of the iterated IRS method, Journal of Sound and Vibration, 211, 123-132, DOI: 10.1006/jsvi.1997.1368

18. GuYAn R.J., 1965, Reduction of stiffness and mass matrices, AIAA Journal, 3, 380

19. Henshell R.D., Ong J.H., 1974, Automatic masters for eigenvalue economization, Earthquake Engineering and Structural Dynamics, 3, 375-383, DOI: 10.1002/eqe.4290030408

20. Jin Y., 2011, Surrogate-assisted evolutionary computation: Recent advances and future challenges, Swarm and Evolutionary Computation, 1, 61-70, DOI: 10.1016/j.swevo.2011.05.001

21. Kim K.-O., Choi Y.-J., 2000, Energy method for selection of degrees of freedom in condensation, AIAA Journal, 38, 1253-1259, DOI: 10.2514/2.1095

22. Lapeer R.J., Gasson P.D., Karri V., 2010, Simulating plastic surgery: From human skin tensile tests, through hyperelastic finite element models to real-time haptics, Progress in Biophysics and Molecular Biology, 103, 208-216, DOI: 10.1016/j.pbiomolbio.2010.09.013

23. LEUNG Y.-T., 1979, An accurate method of dynamic substructuring with simplified computation, International Journal for Numerical Methods in Engineering, 14, 1241-1256, DOI: 10.1002/nme.1620140809

24. Levy R., 1971, Guyan reduction solutions recycled for improved accuracy, Technical Report No. 19710026790, NASTRAN: Users Experiences, NASA, Langley Res. Center

25. Li W., 2003, A degree selection method of matrix condensations for eigenvalue problems, Journal of Sound and Vibration, 259, 409-425, DOI: 10.1006/jsvi.2002.5336 
26. Matta K.W., 1987, Selection of degrees of freedom for dynamic analysis, Journal of Pressure Vessel Technology, 109, 65-69, DOI: 10.1115/1.3264857

27. Mokeyev V.V., 1998, A frequency condensation method for the eigenvalue problem, Communications in Numerical Methods in Engineering, 14, 1-8

28. Mrozek A., Kuś W., Burczyński T., 2015, Nano level optimization of graphene allotropes by means of a hybrid parallel evolutionary algorithm, Computational Materials Science, 106, 161-169, DOI: 10.1016/j.commatsci.2015.05.002

29. Mucha W., 2019, Application of artificial neural networks in hybrid simulation, Applied Sciences, 9, DOI: $10.3390 /$ app9214495

30. Mucha W., Kuś W., 2017, Application of mode superposition to hybrid simulation using real time finite element method, Mechanika, 23, 673-677

31. Mucha W., Kuś W., 2018, Mountain bicycle frame testing as an example of practical implementation of hybrid simulation using RTFEM, [In:] Computer Methods in Mechanics (CMM2017). Proceedings of the 22nd International Conference on Computer Methods in Mechanics, Lublin, Poland, 2017, AIP Conference Proceedings, 1922, AIP Publishing, Melville, 140002-1-140002-9

32. ONG J.H., 1987, Improved automatic masters for eigenvalue economization, Finite Elements in Analysis and Design, 3, 149-160, DOI: 10.1016/0168-874X(87)90006-0

33. Popplewell N., Bertels A.W.M., Arya B., 1973, A critical appraisal of the elimination technique, Journal of Sound and Vibration, 31, 213-233, DOI: 10.1016/S0022-460X(73)80376-1

34. Poteralski A., Szczepanik M., Beluch W., Burczyński T., 2014, Optimization of composite structures using bio-inspired methods, [In:] Artificial Intelligence and Soft Computing, L. Rutkowski, M. Korytkowski, R. Scherer, R. Tadeusiewicz, L.A. Zadeh, J.M. Zurada (Eds.), Springer International Publishing, 385-395

35. Qu Z.Q., 2013, Model Order Reduction Techniques with Applications in Finite Element Analysis, Springer London

36. Qu Z.-Q., Fu Z.-F., 2000, An iterative method for dynamic condensation of structural matrices, Mechanical Systems and Signal Processing, 14, 667-678, DOI: 10.1006/mssp.1998.1302

37. Ramos M.D.C., Mosqueda G., Hashemi M.J., 2016, Large-scale hybrid simulation of a steel moment frame building structure through collapse, Journal of Structural Engineering, 142, 04015086, DOI: 10.1061/(ASCE)ST.1943-541X.0001328

38. Ramsden J.N., Stoker J.R., 1969, Mass condensation? A semi-automatic method for reducing the size of vibration problems, International Journal for Numerical Methods in Engineering, 1, 333-349, DOI: 10.1002/nme.1620010403

39. Shah V.N., Raymund M., 1982, Analytical selection of masters for the reduced eigenvalue problem, International Journal for Numerical Methods in Engineering, 18, 89-98, DOI: 10.1002/nme.1620180108

40. Suarez L.E., Singh M.P., 1992, Dynamic condensation method for structural eigenvalue analysis, AIAA Journal, 30, 1046-1054

41. Zienkiewicz O.C., TAYlor R.L., 2000, The Finite Element Method, Volume 1: The Basis, 5th ed. Butterworth-Heinemann, Oxford 